Bull. Korean Math. Soc. 45 (2008), No. 4, pp. 797-800

\title{
AN ANDERSON'S THEOREM ON NONCOMMUTATIVE RINGS
}

\author{
Chan Huh, Nam Kyun Kim, and Yang Lee
}

\begin{abstract}
Let $R$ be a ring and $I$ be a proper ideal of $R$. For the case of $R$ being commutative, Anderson proved that $(*)$ there are only finitely many prime ideals minimal over $I$ whenever every prime ideal minimal over $I$ is finitely generated. We in this note extend the class of rings that satisfies the condition $(*)$ to noncommutative rings, so called homomorphically IFP, which is a generalization of commutative rings. As a corollary we obtain that there are only finitely many minimal prime ideals in the polynomial ring over $R$ when every minimal prime ideal of a homomorphically IFP ring $R$ is finitely generated.
\end{abstract}

Throughout every ring is associative with identity unless otherwise stated. The $n$ by $n$ matrix ring over a ring $R$ is denoted by $\operatorname{Mat}_{n}(R)$. Due to Bell [2], a right (or left) ideal $I$ of a ring $R$ is said to have the insertion-of-factors-property (simply $I F P$ ) if $a b \in I$ implies $a R b \subseteq I$ for $a, b \in R$. A ring $R$ is called IFP if the zero ideal of $R$ has the IFP. For a ring $R$ and an ideal $I$, note that $I$ has the IFP if and only if $R / I$ is an IFP ring. A ring is called abelian if each idempotent is central. IFP rings are abelian by a simple computation. Shin [9] used the term $S I$ for the IFP; while IFP rings are also known as semicommutative in Narbonne's paper [8]. $r_{R}(-)$ (resp. $\left.\ell_{R}(-)\right)$ is used for the right (resp. left) annihilator in a ring $R$.

Proposition 1. For a ring $R$ the following conditions are equivalent:

(1) $R$ is IFP;

(2) Any left annihilator in $R$ is an ideal;

(3) Any right annihilator in $R$ is an ideal;

(4) $R / r_{R}(A)$ is an IFP ring for any $A \subseteq R$;

(5) $R / \ell_{R}(A)$ is an IFP ring for any $A \subseteq R$.

Received April 24, 2008.

2000 Mathematics Subject Classification. 16D25, 16N60.

Key words and phrases. commutative ring, (homomorphically) IFP ring, minimal prime ideal.

The first author was supported for two years by Busan National University Research Grant. The second named author was supported by LG Yonam Foundation in the program year 2006. The third named author was supported by the Korea Research Foundation Grant funded by the Korean Government (KRF-2005-015-C00011). 
Proof. The equivalences of (1), (2) and (3) are obtained from the definition. $(4) \Rightarrow(1)$ and $(5) \Rightarrow(1)$ are shown by the case of $A=R$. (1) $\Rightarrow(4)$ is proved by the proof of $[9$, Proposition $1.6(1)]$. (1) $\Rightarrow(5)$ is similar to $(1) \Rightarrow(4)$.

A ring is called reduced if it has no nonzero nilpotent elements. Reduced rings and commutative rings are both IFP, but the converses need not hold by [6, Proposition 1.2].

Based on Proposition 1(4), (5) we introduce the following concept: a ring $R$ is called homomorphically IFP if $R / I$ is IFP for every proper ideal $I$ in $R$. Homomorphically IFP rings are clearly IFP but the converse does not hold in general by Example 2(1) below. The class of (homomorphically) IFP rings is closed by direct products. A ring is called right (resp. left) duo if every right (resp. left) ideal is two-sided; a ring is called duo if it is both right and left duo. Commutative rings are clearly duo, but there exist many left or right duo rings which are noncommutative in $[3,4]$. Left or right duo rings are homomorphically IFP by Proposition 1, but there may exist many homomorphically IFP rings which are neither left nor right duo by Example 2(2) below. In the following we see the relations among IFP rings, homomorphically IFP rings, commutative rings, and domains.

Example 2. (1) Domains need not be homomorphically IFP. The $\operatorname{ring} R=$ $\mathbb{Z}+\mathbb{Z} i+\mathbb{Z} j+\mathbb{Z} k$ in $[5$, Exercise $2 \mathrm{~A}]$ is the ring of quaternions with integer coefficients, so it is a domain. But $R / p R$ is isomorphic to the $\operatorname{Mat}_{2}\left(\mathbb{Z}_{p}\right)$ by the argument in $[5$, Exercise $2 \mathrm{~A}]$, where $p$ is any odd positive integer and $\mathbb{Z}_{p}$ is the field of integers modulo $p . \operatorname{Mat}_{2}\left(\mathbb{Z}_{p}\right)$ is non-abelian and so is not (homomorphically) IFP.

(2) Simple domains are clearly homomorphically IFP. There are many noncommutative simple domains, e.g. $n$th Weyl algebras over any field of characteristic zero by [7, Theorem 3.5], but these are neither left nor right duo.

We now generalize the Anderson's result [1, Theorem] to homomorphically IFP rings.

Theorem 3. Let $R$ be a homomorphically IFP ring and $I$ be a proper ideal of $R$. If every prime ideal minimal over I is finitely generated then there are only finitely many prime ideals minimal over $I$.

Proof. Let $S=\left\{P_{1} \cdots P_{n} \mid\right.$ each $P_{i}$ is a prime ideal minimal over $I$ and $n=$ $1,2, \ldots\}$. If some element of $S$ is contained in $I$, then there are only finitely many prime ideals minimal over $I$ obviously. So assume that $C \nsubseteq I$ for each $C \in S$. According to the proof of [1, Theorem], consider the set $T=\{J \mid J$ is an ideal of $R$ with $J \supseteq I$ and $C \nsubseteq J$ for each $C \in S\}$, partially ordered by $\subseteq$. Take a chain $U=\left\{K_{a} \mid a \in A\right\}$ in $T$ and put $K=\cup_{a \in A} K_{a}$. We will show $K \in T$. Assume on the contrary that there exists $C=P_{1} \cdots P_{n} \in S$ with $C \in K$. Each $P_{i}$ is finitely generated by hypothesis, say $P_{i}=\sum_{t=1}^{m_{i}} R p(i)_{t} R$ for $i=1, \ldots, n$. Then every $p(1)_{b_{1}} \cdots p(n)_{b_{n}} \in C$ is contained in $K$ where 
$p(i)_{b_{i}} \in P_{i}$, and the cardinality of $V=\left\{p(1)_{b_{1}} \cdots p(n)_{b_{n}} \mid p(i)_{b_{i}} \in P_{i}\right\}$ is less than or equal to $m_{1} \cdots m_{n}$. Since $V \subseteq K$ and $V$ is finite, there exists $K_{a} \in U$ with $V \subseteq K_{a}$. Now since $R$ is homomorphically IFP, $R / K_{a}$ is an IFP ring; hence $p(1)_{b_{1}} \cdots p(n)_{b_{n}} \in K_{a}$ implies $p(1)_{b_{1}} R \cdots R p(n)_{b_{n}} \subseteq K_{a}$, entailing $C=\left(\sum_{t=1}^{m_{1}} R p(1)_{t} R\right) \cdots\left(\sum_{t=1}^{m_{n}} R p(n)_{t} R\right) \subseteq K_{a} \in T$. This is a contradiction, and so $K \in T$. Then there exists a maximal element $Q$ in $T$. Next assume that there are $a, b \in R$ such that $a R b \subseteq Q$ and $a \notin Q, b \notin Q$. Then by the maximality of $Q$, there exist $C_{1}, C_{2} \in S$ such that $C_{1} \subseteq Q+R a R$ and $C_{2} \subseteq Q+R b R$. But $C_{1} C_{2}=(Q+R a R)(Q+R b R) \subseteq Q$ and $C_{1} C_{2} \in S$, a contradiction. Thus $Q$ is a prime ideal of $R$ with $I \subseteq Q$, and so by [5, Proposition 2.3] there exists a prime ideal $P$ minimal over $I$ with $P \subseteq Q$. Then $P \in S$ with $P \subseteq Q \in T$, a contradiction.

We obtain the following from Theorem 3, letting $I=0$.

Corollary 4. Let $R$ be a homomorphically IFP ring. If every minimal prime ideal of $R$ is finitely generated, then there are only finitely many minimal prime ideals in $R$.

Given a ring $R$ the polynomial ring, with a set $X$ of commuting indeterminates (possibly infinite) over $R$, is denoted by $R[X]$. We next observe minimal prime ideals of polynomial rings over homomorphically IFP rings.

Corollary 5. Let $R$ be a homomorphically IFP ring. If every minimal prime ideal of $R$ is finitely generated, then there are only finitely many minimal prime ideals in $R[X]$.

Proof. We first recall the following well-known facts: (i) $P$ is a (minimal) prime ideal of $R$ if and only if $P[X]$ is a (minimal) prime ideal of $R[X]$, (ii) $Q \cap R$ is a prime ideal of $R$ for any prime ideal $Q$ of $R[X]$. By (i) and (ii), each minimal prime ideal of $R[X]$ is of the form $P[X]$ for some minimal prime ideal $P$ of $R$. If every minimal prime ideal of $R$ is finitely generated then there are only finitely many minimal prime ideals in $R$ by Corollary 4 . Thus $R[X]$ has only finitely many minimal prime ideals by the argument above.

\section{References}

[1] D. D. Anderson, A note on minimal prime ideals, Proc. Amer. Math. Soc. 122 (1994), no. $1,13-14$

[2] H. E. Bell, Near-rings in which each element is a power of itself, Bull. Austral. Math. Soc. 2 (1970), 363-368.

[3] A. W. Chatters and W. Xue, On right duo p.p. rings, Glasgow Math. J. 32 (1990), no. $2,221-225$.

[4] R. C. Courter, Finite-dimensional right duo algebras are duo, Proc. Amer. Math. Soc. 84 (1982), no. 2, 157-161.

[5] K. R. Goodearl and Jr. R. B. Warfield, An introduction to Noncommutative Noetherian Rings, London Mathematical Society Student Texts, 16. Cambridge University Press, Cambridge, 1989. 
[6] N. K. Kim and Y. Lee, Extensions of reversible rings, J. Pure Appl. Algebra 185 (2003), no. $1-3,207-223$.

[7] J. C. McConnell and J. C. Robson, Noncommutative Noetherian Rings, A WileyInterscience Publication, John Wiley \& Sons, Ltd., Chichester, 1987.

[8] L. M. de Narbonne, Anneaux semi-commutatifs et uniseriels; anneaux dont les ideaux principaux sont idempotents, [Semicommutative uniserial rings; rings whose principal ideals are idempotent] Proceedings of the 106th National Congress of Learned Societies (Perpignan, 1981), 71-73, Bib. Nat., Paris, 1982.

[9] G. Shin, Prime ideals and sheaf representation of a pseudo symmetric ring, Trans. Amer. Math. Soc. 184 (1973), 43-60.

CHAN HuH

Department of Mathematics

Busan National University

Busan 609-735, Korea

E-mail address: chuh@pusan.ac.kr

NAm Kyun Kim

College of Liberal ArTs

Hanbat National University

DAEJEON 305-719, Korea

E-mail address: nkkim@hanbat.ac.kr

YANG LEE

Department of Mathematics Education

Busan National University

BUSAN 609-735, Korea

E-mail address: ylee@pusan.ac.kr 